\title{
A hybrid scheduling approach for automated flowshops with material handling and time constraints
}

\author{
Adrián M. Aguirre ${ }^{\mathrm{a}}$, Carlos A. Méndez ${ }^{\mathrm{a} *}$ and Pedro M. Castro ${ }^{\mathrm{b}}$ \\ ${ }^{a}$ INTEC (UNL-CONICET), Santa Fe, Argentina; ${ }^{b} U M O S E / L N E G$, Lisboa, Portugal
}

(Received 17 December 2012; accepted 15 January 2014)

\begin{abstract}
Flowshop scheduling problems have been extensively studied by several authors using different approaches. A typical flowshop process consists of successive manufacturing stages arranged in a single production line where different jobs have to be processed following a predefined production recipe. In this work, the scheduling of a complex flowshop process involving automated wet-etch station from semiconductor manufacturing systems requires a proper synchronisation of processing and transport operations, due to stringent storage policies and fixed transfer times between stages. Robust hybrid solution strategies based on mixed integer linear programming formulations and heuristic-based approaches, such as aggregation and decomposition methods, are proposed and illustrated on industrial-scale problems. The results show significant improvements in solution quality coupled with a reduced computational effort compared to other existing methodologies.
\end{abstract}

Keywords: hybrid decomposition approach; MILP-based strategies; large-scale scheduling problems; semiconductor manufacturing system (SMS); automated wet-etch station (AWS); modelling and optimization

\section{Introduction}

The typical flowshop scheduling problems (FSP) have greatly attracted the attention of the research and industrial community in recent years. In these, jobs are grouped and then performed following a sequence of units under flexible processing times, where every unit can only perform one job at a time. Additionally, the assumption of limited intermediate storage between consecutives machines through the process constitutes the major issue for real-life industrial application problems in this area.

The traditional FSP is usually focused on finding the best processing sequence that minimises the total resident time of all jobs in the system, i.e. makespan. Following this criterion, it is easy to demonstrate that the FSP are NP-hard, due to the nature of the binary decisions needed to achieve the optimal solution to this complex optimisation problem.

In addition, parallel processing machines, set-up times and multiple objectives were already considered in this problem. Thus, different types of solution methods that take into account all these features were reported in the literature. Heuristics and meta-heuristics (Rossi, Pandolfi, and Lanzetta 2013; Seidgar et al. 2014; Wang and Liu 2013), mathematical programming approaches (Cortés, García, and Hernández 2012) and hybrid algorithms (Fu, Sivakumar, and Li 2012; Wu et al. 2012; Ribas, Companys, and Tort-Martorell 2013) were recently developed for this kind of challenging optimisation problem.

The problem addressed in this work considers the typical flowshop scheduling process with the added complexity of transfer operations among the different stages of the system, performed by a single resource. This kind of problems has been classified as automated FSP in the literature (Crama 1997; Gupta and Stafford 2006), and its application appears in several manufacturing areas and industries, such as manufacturing of printed circuit boards in the electroplating plants, commonly known as the hoist scheduling problem (Phillips and Unger 1976; Shapiro and Nuttle 1988; Yin 1994; Zhou and Liu 2008) and also automated wet stations in the wafer fabrication process of semiconductor manufacturing industries (Gupta et al. 2006; Lee, Lee, and Lee 2007; Ham 2012; Kim, Lee, and Lee 2012, 2013).

The complex problem in the automated wet-etch station (AWS; Uzsoy, Lee, and Martin-Vega 1992) considers the scheduling of different jobs (typically a job is a lot of 25 wafers), $i=1,2, \ldots, N$, in several stages (Baths or Units), $j=1,2, \ldots, M$, in a serial flowshop multi-product batch process with mixed intermediate storage (MIS) policies and a

\footnotetext{
*Corresponding author. Email: cmendez@intec.unl.edu.ar 